\title{
PAOLO MANTEGAZZA E GLI UCCELLI DEL CIELO
}

\author{
RENATO MASSA (*)
}

SunTO. - L'interesse di Paolo Mantegazza nei confronti della biologia degli uccelli iniziò con una semplice attenzione al mondo degli allevatori di piccoli uccelli canori. A partire da queste premesse, Mantegazza si interessò dapprima alle ricerche di Riccardo Stradi sui pigmenti delle piume degli uccelli fino al punto di produrre, nel 1998, uno splendido volume-strenna dell'università dedicato proprio a questo tema. Negli stessi anni, egli seguì con il massimo interesse l'istituzione e il funzionamento della Stazione ornitologica di Albonico dove si produssero, oltre a molte tesi di laurea in scienze biologiche e/o naturali, anche diversi lavori di ricerca su particolari aspetti ecologici della vita degli uccelli canori. Il successo della Stazione di Albonico suggerì di tentare di salvare il vecchio zoo di Milano istituendo, sulle orme del Museo e dell'Acquario civici, una Stazione di ricerca e di conservazione legata a uno zoo rinnovato. Il progetto fu approfondito e fortemente appoggiato, dal rettore Paolo Mantegazza, però, uno sfavorevole cambiamento politico dell'amministrazione comunale alla fine degli anni ' 80 troncò decisamente le speranze e vanificò tutto il lavoro fino a quel momento effettuato. La sfortunata vicenda dello zoo milanese fu tuttavia seguita da un'altra di maggiore successo, quella dello studio del linguaggio dei pappagalli africani che, con il pieno appoggio del Rettore, furono da un lato ospitati in una struttura costruita ad hoc nel cortile del dipartimento di Farmacologia, dall'altro studiati anche in natura in una serie di brevi spedizioni in Tanzania e Uganda. I risultati di questa attività sono riassunti in una ventina di pubblicazioni scientifiche, in un libro tecnico e in un libro divulgativo. Una nota a parte merita la vicenda della lista dei nomi italiani degli uccelli di tutto il mondo, un progetto iniziato più che altro come gioco semantico sul quale poi ci si appassionò tanto da osare di richiedere un contributo per la sua pubblicazione al Rettorato, contributo che fu generosamente concesso. Il risultato ottenne inaspettatamente addirittura una lode della Accademia della Crusca e fu ampiamente utilizzato negli anni successivi nella editoria naturalistica divulgativa. Dopo la prima edizione del 1993 ne comparve una revisione nel 2000 e più recentemente una versione online arricchita da capitoli introduttivi ai diversi gruppi sistematici nonché di molte immagini scattate da fotografi giramondo. In definitiva, Paolo Mantegazza si interessò personalmente di biologia degli uccelli e appoggiò concretamente molte iniziative di ricerca e cultura nel merito nell'ambito della Università degli Studi di Milano.

(*) Dipartimento di Biologia, Università degli Studi di Milano, Italia. E-mail: renato.j.massa@gmail.com 
$* * *$

ABSTRACT. - The earliest interest of Paolo Mantegazza in bird biology was expressed as an attention to the world of ornamental bird breeders. Later on, Mantegazza was so deeply fascinated by Riccardo Stradi's research on bird feather's pigments to sponsor a magnificent University annual book just dedicated to this theme. At the same time he became very much interested and openly supported the establishment of a new ornithological station at Albonico, a supporting institution that readily produced several degree theses in biological and /or natural sciences and, in addition, several research papers on special ecological themes regarding . the life history of song birds. The success of the above cited station also suggested to try to save the old Milan zoo by establishing a zoo research station on the model of the corresponding institutions of the Milan aquarium and natural history museum. The project was discussed in comparison with the Jersey Wildlife Preservation Trust, and, as such, was strongly supported by Mantegazza, but due to a political change of the Miano city hall administration, it was suddenly rejected while the whole work done up to that point was completely lost. The unfortunate story of the Milan zoo was shortly followed by a much luckier one, regarding the study of the parrot language. With the moral and material help of Mantegazza, a number of African parrots were even hosted in an especially built glass structure within the domain of the Department of Pharmacology. In addition, they were also studied on the field in a series of short expeditions to Tanzania and Uganda. The results of these activities were summarized in a numbers of papers and books. A special memory is also worth about the world list of the Italian bird names, a project that was started almost as a semantic joke and was subsequently so much developed to suggest to ask to our Rector (and readily obtain) a University contribution for its publication. The resulting bird name list was even worth a Crusca Academy praise and was extensively adopted since its publication in the popular ornithological literature in the Italian language. The first paper print, delivered in 1993, was followed by a second paper print in the year 2000 and, more recently, by an electronic version enriched by introductory texts to orders and families as well as by several pictures by a number of photographers. In summary, Paolo Mantegazza was deeply interested in bird biology and encouraged/sponsored a number of researches and cultural events on this topic within the University of Milano.

\section{INTRODUZIONE}

L'istituto di Farmacologia dell'Università degli studi di Milano fu, ed è tuttora, un'istituzione di enorme importanza che produsse o influenzò in diversi modi molti personaggi chiave della ricerca medica e biologica italiana. Io, che ebbi la fortuna di operarvi tra il 1968 e il 1983, tra tutti coloro che ebbi l'onore di conoscere personalmente, rimasi legato in massimo grado - oltre che al professor Luciano Martini, mio capo e maestro, - anche a un'altra persona straordinaria che a quei tempi era un semplice professore ordinario e che, dopo diversi anni, divenne dapprima preside della facoltà di Medicina e poi anche rettore dell'università, e mantenne questa carica per ben diciassette anni per un 
totale di sei mandati, venendo sempre rieletto a larga maggioranza. Parlo naturalmente del professor Paolo Mantegazza.

La mia presentazione al professor Mantegazza fu decisamente insolita dato che fu fatta dal portinaio dell'Istituto, un simpatico signore baffuto che rispondeva al cognome di Pirovano e che teneva una piccola voliera con un gruppetto di bei canarini in un locale a vetri nascosto dietro l'aula situata all'ingresso, dove si svolgevano, tra l'altro, molte conferenze di relatori esterni invitati nell'istituto.

Il professor Paolo Mantegazza, oltre che essere importante farmacologo, ebbe anche molti altri interessi e, tra l'altro, fu un autentico appassionato di ornitologia, inizialmente interessato soprattutto all'allevamento di cardellini e di specie affini ma poi via via allargando e approfondendo i suoi interessi scientifici e culturali. A quei tempi, usava spesso invitare il portinaio dell'istituto, signor Pirovano a prendere il caffè con lui al bar per parlare di uccelli. Un giorno cercò il portinaio mentre questi, dietro mia richiesta, mi stava illustrando gli uccelli della sua voliera, e così trovò anche me. Pirovano mi presentò brevemente a Mantegazza come nuovo borsista presso il gruppo di endocrinologia del professor Luciano Martini.

Notai subito che il professor Paolo Mantegazza era persona semplice e cordiale ma che la serena autorità che emanava da lui si imponeva comunque con un flusso tanto forte e tanto convincente da rendere assolutamente impossibile fraintendere il senso della sua cordialità. Più tardi mi accorsi anche che ascoltava tutti e che era ugualmente capace di affrontare un dialogo difficile con un ministro arrogante o con un rappresentante degli studenti pieno di idee bislacche. Per questo, egli divenne per me un forte riferimento etico nel corso dei lunghi anni in cui ci trovammo a operare insieme, nei nostri rispettivi ruoli e livelli, all'interno della Università degli studi di Milano. Devo anche dire che nel corso dei regolari incontri che cercavo di avere con lui per tenerlo al corrente dello sviluppo del mio lavoro ricevetti sempre non solo un vivo incoraggiamento ma anche suggerimenti concreti e spesso copie di pubblicazioni pertinenti.

\section{I COLORI DELLE PIUME}

Nei primi tempi del nostro lungo rapporto di profonda e, da parte mia, filiale amicizia, le pause caffè con il professor Mantegazza e il 
signor Pirovano mi confortarono anche umanamente, facendomi pensare che la mia passione per il mondo degli uccelli si potesse ritrovare in una congerie abbastanza vasta e varia di persone, ivi comprese alcune di esse altamente rispettabili.

Tra queste illustri persone debbo ora ricordare almeno il professor Riccardo Stradi, docente di chimica analitica che, vivamente incoraggiato da Mantegazza e modestamente anche dal sottoscritto, intraprese l'avventura dello studio dei pigmenti delle piume degli uccelli, studio che gli valse ben presto notevoli pubblicazioni scientifiche su riviste di valore (1-23) nonché una sicura reputazione internazionale che lo stesso Mantegazza volle sottolineare e celebrare con una prestigiosa pubblicazione dell'università, curata graficamente in modo magnifico dalla signora Grazia Buccellati.

\section{ENDOCRINOLOGIA AVIARIA}

Quanto a me, i miei primi lavori sul tema degli uccelli, in accordo con la mia collocazione accademica del momento, furono di carattere endocrinologico (24-34). Di particolare importanza per la mia formazione scientifica fu il periodo trascorso in Scozia, tra il 1979 e il 1981, presso il laboratorio del Roslin Institute diretto dal professor Peter J. Sharp. Qui studiai il ruolo degli ormoni steroidei nel ciclo riproduttivo degli uccelli, argomento che interessava non solo la storia naturale ma anche la produzione di uova e di carne dell'industria avicola.

\section{La Stazione ornitologica di AlbOnico}

Poco dopo, non appena rientrato in patria, mi occupai di mettere in piedi ad Albonico, in un'area collinare a nord del lago di Como, una stazione ornitologica dedicata allo studio delle migrazioni. Nel ricordare questa impresa, che ci accompagnò per un intero decennio, devo ricordare il dottor Vittorio Vigorita, il funzionario della regione Lombardia responsabile di questo settore, che letteralmente si entusiasmò al progetto e lo sostenne per alcuni anni. Non è certo qui il caso di ricordare tutto il lungo e complesso lavoro di quel decennio e le molte tesi di laurea degli studenti coinvolti ma vorrei almeno ricordare le osservazioni sulla migrazione a tappe del pettirosso e i vari lavori sulla frenetica attività riprodut- 
tiva dell'averla piccola nel breve periodo della sua permanenza in Europa (maggio-agosto) tra un soggiorno africano e l'altro (35-42).

\section{La battaglia PeRduta PER lo ZOO Di Milano}

Il grande problema che ci si presentò alla fine degli anni ' 80 fu tuttavia un altro che riguardava il futuro di una storica istituzione cittadina, lo zoo di via Manin che era piccolo, sacrificato, pieno di limiti ma pur sempre visitato da centinaia di migliaia di cittadini alla ricerca di qualche brandello di naturalità. Quando il Comune di Milano incominciò a interrogarsi circa il possibile futuro del suo piccolo zoo cittadino, il nostro rettore sposò senza esitazione il nostro impegno in favore della sua conservazione ed evoluzione verso un nuovo e più interessante oggetto culturale. In questa generosa battaglia si aggiunse ben presto quale autorevole alleato il direttore del dipartimento di Biologia, prof. Giulio Lanzavecchia, che, a fronte dell'interesse anche concreto del Rettore, accettò di organizzare anche un convegno ad hoc che avrebbe dovuto chiarire agli amministratori cittadini l'interesse del mondo scientifico nei confronti di una istituzione ostensiva di animali vivi che si impegnasse in prima linea anche nella ricerca scientifica e nella conservazione biologica.

Non posso certo rievocare tutte le vicende di quella difficile battaglia, ma non posso dimenticare la giornata di studio nella quale si riuscì a riunire a Milano, presso un'aula della sede centrale dell'Università degli Studi, la maggior parte degli zoologi italiani che si occupavano di questo settore dei nostri studi e inoltre un rappresentante del Jersey Wildlife Preservation Trust, la fondazione di Jersey che gestisce uno zoo dove vengono riprodotte in cattività unicamente le specie in immediato pericolo di estinzione. Parve, nell'euforia straordinaria di quella giornata, di essere ormai vicinissimi al nostro obiettivo, ma poi le lentezze, le storture e i calcoli meschini della politica in breve tempo capovolsero la situazione e lo zoo di Milano scomparve semplicemente nel nulla. Che dire? D'altra parte, da quei giorni a oggi la politica non è certamente migliorata.

\section{I PAPPAGALLI AFRICANI}

Alla vicenda dello zoo milanese se ne sovrappose un'altra che per fortuna fu di maggiore successo, quella dei pappagalli africani dei quali 
io personalmente avevo incominciato a occuparmi alla fine degli anni '80 con diversi programmi di studi etologici, bioacustici e anche sistematici. I primi pappagalli che raccogliemmo furono un dono del direttore dello zoo, Arduino Terni, e presso lo zoo furono anche sistemati. Tuttavia, i programmi di studio erano appena agli esordi quando la giunta comunale decise semplicemente di ignorarli e di chiudere lo zoo e ci inviò anche una lettera intimandoci lo sgombero degli uccelli.

In quella occasione, il Rettore Paolo Mantegazza, che stava attentamente seguendo il nostro lavoro, che aveva sponsorizzato il convegno e che stava letteralmente sognando insieme con noi un futuro alternativo delle istituzioni culturali cittadine di carattere biologico ci propose di allestire una semplice struttura per ospitare la collezione di uccelli vivi ignorata e disprezzata dalla giunta pro tempore del comune di Milano. Era una bella serra lunga una ventina di metri e larga forse cinque, che venne rapidamente montata nel cortile del dipartimento di Farmacologia e che ben presto divenne il teatro di una assidua attività di ricerca specializzata sui pappagalli africani. Ci occupammo di vari aspetti della loro biologia ma in modo particolare di bioacustica, con l'obiettivo di analizzare e interpretare i significati semantici delle loro diverse vocalizzazioni e imitazioni, suoni di distress, suoni di interazione di coppia e suoni di interazione parentale con i pulcini. Inoltre, riuscimmo anche a studiare i tratti essenziali dell'evoluzione di questo gruppo di uccelli mediante un semplice approccio biochimico (43-55).

Desiderando poi rispondere alla inevitabile (seppure ingenua) domanda di qualcuno sulla validità scientifica di uno studio sui pappagalli condotto interamente in condizioni di cattività, nel febbraio del 1993 organizzammo una spedizione in Tanzania per studiare le vocalizzazioni di alcune coppie di pappagallo dal ventre arancio durante il periodo riproduttivo. I risultati di questi lavori consistono in una ventina di pubblicazioni scientifiche e comunicazioni a convegni e inoltre in un libro divulgativo che racconta la nostra appassionante esperienza in Tanzania.

Continuammo poi l'entusiasmante esperienza tanzaniana anche in Uganda, lavorando ancora una volta sia in laboratorio sia sul campo, in questo caso sul pappagallo cenerino e sulla sua capacità di comporre musica a partire dal semplice insegnamento delle note fondamentali. In Uganda, poi, organizzammo anche una collaborazione pluriennale con la locale Makerere University, ma questo è un altro discorso che ormai esula dalla comunicazione che oggi ho l'onore di tenere in questa sede. 
Una nota a parte merita la vicenda della lista dei nomi italiani degli uccelli di tutto il mondo, un progetto da me iniziato più che altro come passatempo semantico sul quale poi mi appassionai tanto da osare di richiedere un contributo per la sua pubblicazione al Rettorato, contributo che mi fu generosamente concesso. Il risultato ottenne una lode della Accademia della Crusca e fu ampiamente utilizzato negli anni successivi nella editoria naturalistica divulgativa. Dopo la prima edizione del 1993 ne comparve una revisione nel 2000 e più recentemente una versione online arricchita da capitoli introduttivi ai diversi gruppi sistematici nonché di immagini di fotografi giramondo (56-58).

\section{GLI UCCELLI DEL CIELO}

Carissimo Rettore, oggi sono felice di poterti pubblicamente ringraziare di tutto quanto hai fatto per noi, per la ricerca che abbiamo avuto la fortuna e l'onore di portare avanti, per la conoscenza delle menti degli animali che anche Tu amavi e ammiravi. Grazie anche della fiducia che hai voluto riporre in noi, fiducia che sempre abbiamo cercato, nei limiti delle nostre modeste forze, di non deludere. Questa fiducia si trasformò a poco a poco in una profonda amicizia e un giorno, in una tristissima circostanza, parlando del dolore nel mondo che entrambi avevamo ben conosciuto, ti domandai accorato come si potesse avere la tua forte e ammirevole fede in Dio. Tu mi rispondesti, con l'umiltà e la semplicità che sempre ti contraddistinse: "È una speranza".

\section{RINGRAZIAMENTI}

Sono molto grato al professor Luciano Martini che, invitandomi a partecipare al convegno dedicato al prof. Paolo Mantegazza, mi ha dato l'occasione di rievocare la sua attiva passione ornitologica e la sua grande figura di Maestro. 


\section{BIBLIOGRAFIA}

1. Hudon J, Wiebe KL, Pini E, Stradi R. Plumage pigment differences underlying the yellow-red differentiation in the Northern Flicker (Colaptes auratus). Comparative Biochemistry and Physiology Part B. Biochemistry and Molecular Biology 01/2015; 183.

2. Hudon J, Storni A, Pini E, Anciães M, Stradi R. Rhodoxanthin as a characteristic keto-carotenoid of manakins (Pipridae). The Auk 01/2012; 129:491-499.

3. Rigano L, Picardo M, Pini E, Stradi R, Meloni M, Bertelli A, Benedusi A, Giuliani G. Novel retinol-like actives from parrots feathers. International Journal of Cosmetic Science 12/2009; 31(6):477-478.

4. Hudon J, Anciães M, Bertacche V, Stradi R. Plumage carotenoids of the Pin-tailed Manakin (Ilicura militaris): evidence for the endogenous production of rhodoxanthin from a colour variant. Comparative Biochemistry and Physiology Part B Biochemistry and Molecular Biology 147(3):402-11. July 2007.

5. Briganti S, Fiori E, Camera E, Pini E, Stradi R, Benedusi A, Giuliani G, Bertelli A, Picaro M. "Novel pigments from parrot feathers with anti-oxidant and photoprotective actions". Comparative Biochemistry and Physiology Part B Biochemistry and Molecular Biology 07/2007; 147(3):402-11. Pigment Cell Research, 20 (5), 450, 2007.

6. Remeš V, Krist M, Bertacche V, Stradi R. Maternal carotenoid supplementation does not affect breeding performance in the Great Tit (Parus major). Functional Ecology 04/2007; 21(4):776-783.

7. McGraw KJ, Hill GE, Stradi R, Parker RS. The effect of dietary carotenoid access on sexual dichromatism and plumage pigment composition in the American goldfinch, Comparative Biochemistry and physiology, PartB: Biochemistry \& Molecular Biology, 131B(2), 261-269, 2002.

8. Saino N, Bertacche V, Ferrari RP, Martinelli R, Møller AP, Stradi R. Carotenoid concentration in barn swallow eggs is influenced by laying order, maternal infection and paternal ornamentation. Proceedings of the Royal Society B: Biological Sciences 09/2002; 269(1501):1729-33.

9. Inouye CY, Hill GE, Stradi R, Montgomerie R. Carotenoid pigments in male house finch plumage in relation to age, subspecies and ornamental coloration. The Auk 10/2001; 118(4).

10. Stradi $\mathrm{R}$, et al.The influence of carotenoid acquisition and utilization on the maintenance of species-typical plumage pigmentation in male American golfinches (Carduelis tristis) andnorthern cardinalis (Cardinalis cardinalis). Physiol BiochemZool. 74(6): 843-852 (2001).

11. Massa R, Stradi R. Colori in volo. Hoepli Ed. 2001.

12. Stradi R, Pini E, Celentano G. The chemical structure of the pigments in Ara macao plumage. Comparative Biochemistry and Physiology Part B Biochemistry and Molecular Biology 09/2001; 130(1):563.

13. Stradi R, Pini E, Celentano G. Carotenoids in bird plumage: the complement of red pigments in the plumage of wild and captive bullfinch (Pyrrhula pyrrhula). 
Comparative Biochemistry and Physiology Part B Biochemistry and Molecular Biology 04/2001; 128(3):529.

14. Saino N, Stradi R, Ninni P, Pini E, Møller AP. Carotenoid Plasma Concentration, Immune Profile, and Plumage Ornamentation of Male Barn Swallows (Hirundo rustica). The American Naturalist 11/1999; 154(4):441-448.

15. Stradi R, Hudon J, Celentano G, Pini E. Carotenoids in bird plumage: the complement of yellow and red pigments in true woodpeckers (Picinae). Comparative biochemistry and physiology. B, Comparative biochemistry 06/1998; 120(2):223-230.

16. Stradi R. The Colour of flight. Carotenoids in bird plumage, Solei press, 1998; 117(1).

17. Stradi R, Rossi E, Celentano G, Bellardi B. Carotenoids in bird plumage: The pattern in three Loxia species and in Pinicola enucleator. Comparative Biochemistry and Physiology Part B Biochemistry and Molecular Biology 02/1996; 113(2-113):427-432.

18. Stradi R, Celentano G, Nava D. Separation and identification of carotenoids in bird's plumage by high-performance liquid chromatography-diode-array detection. Journal of chromatography. B, Biomedical applications 09/1995; 670(2):337-48.

19. Veronelli M, Zerbi G, Stradi R. In situ resonance Raman spectra of carotenoids in bird's feathers. J. Raman Spectrosc., 26 (8\&9), 683-92 (1995).

20. Stradi R, Celentano G, Rossi E, Rovati G, Pastore M. Carotenoids in bird plumage-I. The carotenoid pattern in a series of palearctic carduelinae. Comparative biochemistry and physiology. B, Comparative biochemistry 01/1995; 110(1-110):131-143.

21. Massa R, Canali G. Il colore in avicoltura. In "Colori in volo" a cura di G. Buccellati. Hoepli, Milano, 1999; pp. 89-116.

22. Saino N, Massa R, Moller AP. Il colore degli uccelli e la selezione sessuale. In "Colori in volo" a cura di G. Buccellati. Hoepli, Milano, 1999; pp. 55-88.

23. Massa R, Stradi R. Il colore degli uccelli con gli occhi del passato. In "Colori in volo" a cura di G. Buccellati. Hoepli, Milano, 1999; pp. 1-20.

24. Massa R, Bottoni L. Effect of Steroidal Hormones on the locomotor activity of the male chaffinch (Fringilla coelebs). Monitore Zoologico Italiano, 1986. 21: 69-76.

25. Massa R, Sharp PJ. The photoperiodic control of concentrations of luteinizing hormone in male migratory chaffinches (Fringilla coelebs L). Bollettino di Zoologia, 1987. 54: 251-253.

26. Sharp PJ, Massa R, Bottoni L, Lucini V, Lea RW, Dunn IC, Trocchi V. Photoperiodic and endocrine control of seasonal breeding in Grey Partridge (Perdix perdix). Journal of Zoology (London), 1986, 209: 187-200.

27. Klandorf H, Massa R, Harvey S, Sharp PJ. Reciprocal antagonistic interactions between thyroid and androgenic hormones. Comparat. Endocrinol. (Life Sci. Adv.), 1988. 7: 1-5.

28. Ricossa C, Massa R. The breeding season of the Red-backed shrike, Lanius collurio, in Northern Italy. Monitore Zoologico Italiano, 1988. 22: 299-305.

29. Fornasari L, Bottoni L, Schwabl H, Massa R. Testosterone in the breeding cycle 
of the Red-backed Shrike Lanius collurio. Ethology, Ecology, Evolution, 1991; 3: 193-196.

30. Bottoni L, Massa R, Fornasari L. The migration of the Robin Erithacus rubecula in the central Pre-Alps of Italy. Ringing and Migration, 1991, 12: 48-53.

31. Silverin B, Massa R, Stokkan KA. Gonadal growth and circulating levels of LH in Great Tits from three different latitudes: an experimental study. General and Comparative Endocrinology, 1993; 90: 14-22.

32. Bottoni L, Massa R, Lea RW, Sharp PJ. Mate choice and reproductive success in the Red-legged partridge Alectoris rufa. Hormones and Behaviour, 1993; 27: 308-317.

33. Massa R, Bottoni L, Fornasari L. Site fidelity and population structure of the Red-backed Shrike Lanius collurio in Northern Italy. Ringing \& Migration, 1993; 14: 129-132.

34. Fornasari L, Bottoni L, Sacchi N, Massa R. Home range overlapping and sociosexual relationships of the Red-backed shrike Lanius collurio. Ethology, Ecology, Evolution, 1994. 6: 169-177.

35. Fornasari L, Piazza E, Schubert M, Massa R. A comparison between two different methods of catch for the study of Passerine migration through the Pre-Alps of Lombardy. In "First Conference on Birds wintering in the Mediterranean Region" a cura di A. Farina, 1986; pp. 137-152.

36. Schubert M, Fedrigo A, Massa R. Timing and pattern of the post-breeding migration of some species of Passerines through Lombardy, Northern Italy. Ringing \& Migration, 1986; 7: 15-22.

37. Massa R. Canary. The Cornell Bioacustic Workstation. Version 1.1. Ethology, Ecology, \& Evolution, 1994. 6: 250-252.

38. Massa R, Bottoni L, Fornasari L, Sacchi N. Studies on the socio-sexual and territorial system of the Red-backed shrike Lanius collurio. In R. Josef \& F.E. Lohrer Eds. Shrikes (Laniidae) of the World: Biology and Conservation. Proceedings of the Western Foundation of Vertebrate Zoology, 1995; 6(1): 172-175.

39. Fornasari L, Conte M, Movalli C, Massa R. Uno studio pluriennale sulla biologia riproduttiva dell'averla piccola Lanius collurio. Suppl. Ric. Biol. Selvaggina, 1995; XXII: 87-96.

40. Fornasari L, Kurlavicius P, Massa R. Red backed shrike (Lanius collurio). In The EBCC Atlas of European breeding birds: their distribution and abundance a cura di W.J.M. Hagemeijer \& M.J. Blair. T.\& AD Poyser, London, 1997; pp. 660-661.

41. Favini G, Fornasari L, Bottoni L, Massa R. A video-taping approach to study nestling diet and parental care of Red-backed shrike Lanius collurio. In Shrikes of the world II: conservation implementation a cura di Reuven Yosef \& Fred Lohrer. International Birdwatching Center, Eilat, 1998.

42. Fornasari L, Massa R. Extra pair copulation in a cluster of Red-backed Shrikes. Proceedings of the Second Congress of the European Ornithologists, 1999.

43. Massa R, Galanti V, Bottoni L. Mate choice and reproductive success in the domesticated budgerigar Melopsittacus undulatus. Italian Journal of Zoology, 1996, 63: 243-246. 
44. Massa R. Performance of socio-sexual activity at a common site in the Orangebellied Parrot Poicephalus rufiventris. Ostrich, 1996; 66: 141-142.

45. Cozzi R, Massa R, Panzica G. The NADPH-diaphorase containing system in the brain of the budgerigar (Melopsittacus undulatus). Cell \& Tissue Research, 1996, 287: 101-112.

46. Venuto V, Bottoni L, Massa R. Bioacustical components and possible significance of the wing-display vocalization of the African Orange-bellied parrot Poicephalus rufiventris. Ostrich, 2000; 71: 131-135.

47. Massa R, Sarà M, Piazza MAS, Di Gaetano C, RandazzoM, Cognetti G. A molecular approach to the taxonomy and biogeography of African parrots. It. J. of Zool, 2000; 67: 313-317.

48. Venuto V, Ferraiuolo V, Bottoni L, Massa R. Distress call in six species of African Poicephalus parrots. Ethology, Ecology, Evolution, 2001; 13: 49-68.

49. Venuto V, Massa R, Bottoni L. African parrot vocalization and their functional significance. Ostrich, 2001; Supplement N. 15, pp. 224-228.

50. Massa R. I pappagalli africani. Edizioni FOI, Piacenza, 2001.

51. Massa R, Bottoni L, Taylor S, Venuto V. African parrot vocalisation studies in captivity and in the wild. In "Bird research and breeding" a cura di Claudia Mettke-Hoffmann \& Udo Ganslosser. Filander Verlag, 2002; pp. 71-82.

52. Amuno JB, Massa R, Dranzoa C. Abundance, movements and habitat use by Grey parrots (Psittacus erithacus) in Budongo and Mabira forest reserves, Uganda. Ostrich, 2007; 78:225-231.

53. Massimino D, Masin S, Bani L, Massa R, Dranzoa C. Partial recovery of an African rainforest bird community 35 years after logging. Ethology Ecology and Evolution, 2008; 20: 321-330.

54. Amuno JB, Massa R, Okethowengo G. Some Observations on Nesting African Grey Parrots in Uganda. Rivista Italiana di Ornitologia, 2010. 80: 57-59.

55. Massa R. Il pappagallo dal ventre arancio. Jaca Book, Milano, 2010.

56. Massa R, Bottoni L, Violani C. Lista in lingua italiana degli uccelli di tutto il mondo. Università degli Studi di Milano, 1993.

57. Massa R, Bottoni L, Violani C. I nomi in lingua italiana degli uccelli di tutto il mondo. Università degli studi di Milano, 2000.

58. Massa R. Tutti gli uccelli di tutto il mondo. Ebook di Amazon con Marco Cotti, 2014. 